\title{
EDITORIAL
}

\section{Introducción al Número Especial sobre Restauración Ecológica en la Ecorregión Magallánica Subantártica}

\author{
Osvaldo J. Vidal ${ }^{1}$
}

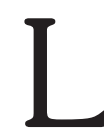

os ecosistemas australes del la ecorregión Magallánica Subantártica forman parte de uno de los biomas mas prístinos del planeta. Estos ecosistemas poseen una biodiversidad única que sustenta altos niveles de endemismos, hábitats y paisajes particulares de un área que, sin embargo, está sometida a una presión antrópica creciente (Rozzi et al. 2012). El rápido desarrollo urbano y turístico que experimenta la región austral de Chile plantea importantes desafíos sobre la conservación y manejo de recursos naturales, dado que paradójicamente, éste desarrollo está asociado a efectos negativos para la biodiversidad. Un paradigmático ejemplo de esta degradación ambiental han sido los sucesivos incendios forestales en el Parque Nacional Torres del Paine (PNTP) (Vidal, 2012). Estos incendios han sido ocasionados por turistas negligentes los que, no respetando las regulaciones del área protegida, han provocado mega-incendios que se han extendido miles de hectáreas. Las consecuencias ecológicas de estos mega-incendios han generando un grave deterioro, incluyendo homogenización biótica, invasiones biológicas y pérdida de hábitats para especies sensibles a los efectos del fuego (Vidal \& Reif, 2011; Vidal, 2012). Eventos como éstos plantean interrogantes sobre acciones plausibles de reparación de los daños ecológicos en sitios perturbados, inspirando este número especial de "Anales del Instituto de la Patagonia" (AIP) sobre restauración ecológica (RE) en la Ecorregión Magallánica Subantártica, con énfasis en Torres del Paine. Por primera vez en Chile, una revista del ámbito académico reúne opiniones de diversos actores nacionales $e$ internacionales para iniciar una discusión sobre RE, una disciplina emergente en Chile. La RE, en su definición mas ampliamente aceptada se refiere al "acto de asistencia artificial a la recuperación de un ecosistema que ha sido dañado por la acción antrópica", cuyo objetivo principal persigue "recuperar la trayectoria histórica de los ecosistemas" (SER, 2004). Esta actividad demanda de la integración, interacción y aplicación de conocimiento de diversas disciplinas como la biología, ecología, agronomía y ciencias forestales, entre muchas otras.

En este número especial de AIP, hemos incluido nueve contribuciones de carácter local y nacional, que describen iniciativas en desarrollo tanto desde el sector productivo como académico. Estas iniciativas dan cuenta de marcos conceptuales y lecciones aprendidas desde la práctica para contextualizar la teoría y el hacer de la RE en la Ecorregión Magallánica Subantártica, con énfasis en el PNTP: Smith-Ramírez et al. (2015) sintetizan las actuales iniciativas desarrolladas en Chile, y que tienen implicancias sobre restauración ecológica a lo largo del país, destacando a la RE como una disciplina de incipiente desarrollo; la contribución de Jaksic \& Fariña (2015), en tanto, ponen en contexto la restauración ecológica en ecosistemas afectados por incendios de Patagonia Austral, concluyendo que el énfasis debería sucederse en bosques incendiados, dado que estos ecosistemas

Laboratorio de Botánica, Instituto de la Patagonia,

Universidad de Magallanes. Avenida Bulnes 01890, Punta Arenas. osvaldo.vidal@umag.cl $\square$ 
experimentarían una retrogresión, en vez de un cambio de estado post-perturbación; Bannister (2015), en tanto, resume las acciones de restauración llevadas a cabo para recuperar los bosques de Ciprés de las Guaitecas (Pilgerodendron uviferum [D. Don] Florin), con recomendaciones concretas aplicables a toda el área de distribución de este ecosistema; Navarro-Cerrillo et al. por su parte, ofrecen un protocolo para la determinación de áreas prioritarias de restauración ecológica a una escala espacial de paisaje. Este estudio de caso, desarrollado con sistemas de información geográfico en PNTP, entroniza criterios como la vegetación pre-incendio, capacidad de respuesta de la vegetación y uso recreativo para definir estas zonas prioritarias; seguidamente, Vidal et al. (2015) presentan una revisión sobre las especies de plantas exóticas y las invasiones biológicas provocadas por éstas, que muestra que las invasiones biológicas en PNTP pueden significar estados críticos para el reestablecimiento de la vegetación nativa; Valenzuela et al. (2015), presentan un modelo conceptual para la rehabilitación forestal en sitios afectados por actividades mineras. Esta aproximación representa una propuesta concreta de rehabilitación cercana a la RE, dando cuenta que los modelos de RE tienen también aplicación en sitios perturbados por actividades productivas extractivas; la contribución de Niculcar et al. (2015) presentan al Banco de Germoplasma del SAG como una herramienta para desarrollar protocolos de re-introducción de especies de plantas sensibles al fuego. Esta publicación da cuenta de la potencial importancia de las instituciones públicas para llevar la $\mathrm{RE}$ a la práctica y de forma masiva; Reppeto \& Cabello, (2015), en tanto, resumen efectos potenciales del turismo en áreas remotas (e.g. senderismo), con sugerencias sobre el manejo de estas prácticas; finalmente Clewell (2015) cierra este número especial con una nota sobre aspectos ecológicos que se deben tener en cuenta al generar iniciativas de RE en ecosistemas dominados por la Lenga (Nothofagus pumilio [Poepp. \& Endl.] Krasser). En una sucinta reflexión, el autor integra los conceptos fundamentales de la RE y los aspectos autoecológicos del bosque de Lenga. Esperamos que este número especial tenga una acogida favorable por la comunidad académica en Chile y con ello, iniciar una discusión sobre esta disciplina emergente que incluya actores de Universidades, Centros de Investigación, Organizaciones Públicas, ONGs, entre otros, con el fin de que la RE en Chile se convierta mas global, mas compleja y mas interdisciplinaria.

\section{LITERATURA CITADA}

Bannister, J. R. (2015). Recuperar bosques no es solo plantar árboles: lecciones aprendidas luego de 7 años restaurando bosques de Pilgerodendron uviferum (D. Don) Florin en Chiloé. Anales del Instituto de la Patagonia, 43(1), 35-51.

Clewell, A. (2015). Ecological restoration principles relative to Nothofagus pumilio (Poepp. \& Endl.) Krasser (Nothofagaceae) forest restoration. Anales del Instituto de la Patagonia, 43(1), 123-126.

Jaksic, F. \& Fariña, J. M. (2015). Incendios, sucesión y restauración ecológica en contexto. Anales del Instituto de la Patagonia, 43(1), 23-34.

Navarro-Cerrillo, R., Olave, F., Hayas, A. \& Castillo, M. (2015). Metodología para la elaboración de un plan de restauración post-incendio en Chile: La experiencia del Parque Nacional Torres del Paine. Anales del Instituto de la Patagonia, 43(1), 53-73.

Niculcar, R., Latorre, K. \& Vidal, O. J. (2015) Conservación ex-situ de plantas en el banco de germoplasma SAG-Magallanes: una herramienta para la restauración ecológica. Anales del Instituto de la Patagonia, 43(1), 109-113.

Repetto, F. \& Cabello, J. L. (2015). Potencial de restauración ecológica en la zona de uso público en el Parque Nacional Torres del Paine. Anales del Instituto de la Patagonia, 43(1), 115-121.

Rozzi, R., Armesto, J. J., Gutiérrez, J. R., Massardo, F., Likens, G. E., Anderson, C. B., Poole, A.,... Arroyo, M. T. K. (2012). Integrating ecology and environmental ethics: Earth stewardship in the southern end of the Americas. BioScience, 62, 226236.

Smith-Ramírez, C., González, M. E., Echeverría, C. \& Lara, A. (2015). Estado actual de la restauración ecológica en Chile, perspectivas 
y desafíos. Anales del Instituto de la Patagonia, 43(1), 11-21.

Society for Ecological Restoration (SER) International Science \& Policy working Group, (2004). The SER International Primer on Ecological Restoration. www. ser.org \& Tucson: Society for Ecological Restoration International.

Valenzuela, P., Arellano, E., Burger, J., Zegers, G. \& Fernández, I. (2015). Bases para el desarrollo de un modelo de rehabilitación forestal en minería utilizando Nothofagus pumilio (Poepp. et Endl.) Krasser. Anales del Instituto de la Patagonia, 43(1), 97-107.

Vidal, O. J. \& Reif , A. (2011). Effect of a touristignited wildfire on Nothofagus pumilio forests at Torres del Paine biosphere reserve, Chile (Southern Patagonia). Bosque, 32, 64-76.

Vidal, O. J. (2012). Torres del Paine, ecoturismo $e$ incendios forestales: Perspectivas de investigación y manejo para una biodiversidad erosionada. Revista Bosque Nativo 50, 33-39.

Vidal, O. J., Aguayo, M., Niculcar, R., Bahamonde, N., San Martín, C., Kusch, A., Latorre, J. \& Félez, J. (2015). Plantas invasoras en el Parque Nacional Torres del Paine (Magallanes, Chile): estado del arte, distribución post-fuego e implicancias en restauración ecológica. Anales del Instituto de la Patagonia, 43(1), 75-96. 
